\title{
The Impact of Miscarriage and Parity on Patterns of Maternal Distress in Pregnancy
}

\author{
Cheryl L. Woods-Giscombé ${ }^{1,{ }^{*}, \text { Marci Lobel }^{2,{ }^{* *}} \text {, and Jamie L. Crandell }}{ }^{1, \dagger}$ \\ ${ }^{1}$ School of Nursing, The University of North Carolina at Chapel Hill, Chapel Hill, North Carolina \\ ${ }^{2}$ Department of Psychology, Stony Brook University, Stony Brook, New York
}

\section{Abstract}

The purpose of the current study was to examine patterns of state anxiety and pregnancy-specific distress across pregnancy in a diverse sample of women with $(n=113)$ and without $(n=250)$ prior miscarriage. For both groups, state anxiety and pregnancy-specific distress were highest in the first trimester and decreased significantly over the course of pregnancy. Compared to women without prior miscarriage, women with prior miscarriage experienced greater state anxiety in the second and third trimesters. Having a living child did not buffer state anxiety in women with a prior miscarriage. Attention to patterns of distress can contribute to delivery of appropriate support resources to women experiencing pregnancy after miscarriage and may help reduce risk for stressrelated outcomes.

\section{Keywords}

miscarriage; pregnancy; anxiety; prenatal stress; perinatal loss

\begin{abstract}
Approximately $20 \%$ of pregnancies in the United States result in miscarriage (Tulandi \& AlFozan, 2009). Miscarriage is a widely used lay term, but there is substantial variability in its definition. Definitions of miscarriage include pregnancy loss prior to viability (Regan \& Rai, 2000; World Health Organization [WHO], 2001), the loss of a fetus weighing less than 500 $\mathrm{g}$ (WHO), and the loss of an embryo or fetus at 20 weeks gestation or less (American College of Obstetrics and Gynecologists [ACOG], 2009). A woman's subjective experience of miscarriage may include any of these definitions. As with other stressful events, the effects of miscarriage vary considerably across individuals (Swanson, Connor, Jolley, Pettinato, \& Wang, 2007), but for many women and their partners, miscarriage is a tragic, complicated, and life-altering experience (Swanson, 1999a). Authors of two extensive reviews concluded that miscarriage results in significant suffering (Frost \& Condon, 1996; Geller, Kerns, \& Klier, 2004). Less is known, however, about how experiences of miscarriage might influence levels of emotional distress during pregnancy following miscarriage. Therefore, the purpose of the current study was to examine patterns of maternal distress across pregnancy in women with and without a history of prior miscarriage.
\end{abstract}

\footnotetext{
(C) 2010 Wiley Periodicals, Inc.

Correspondence to Cheryl L. Woods-Giscombé, School of Nursing, The University of North Carolina at Chapel Hill, Chapel Hill, NC 27599-7460.

*Assistant Professor.

***Professor.

$\dagger$ Research Assistant Professor.
} 
During the last two decades, research on the psychological impact of miscarriage has grown, including studies of women who have experienced miscarriage exclusively and mixedsample studies of various types of perinatal loss including miscarriage, stillbirth, and neonatal death. This body of research has established an empirical foundation for understanding the lived experiences of miscarriage. For example, women who experience miscarriage worry about future pregnancies (Geller et al., 2004) and may perceive a subsequent pregnancy as especially precious and valuable (DeLuca \& Lobel, 1995). Pregnancy after miscarriage can be experienced as emotionally and psychologically distressing (DeBackere, Hill, \& Kavanaugh, 2008; Geller et al., 2004; Tsartsara \& Johnson, 2006).

\section{CONSEQUENCES OF PRENATAL STRESS AND DISTRESS}

Psychological stress and its emotional concomitants, commonly labeled "distress," influence health primarily through health-impairing behaviors and physiological responses that challenge vascular, immune, metabolic, or neuroendocrine functioning (McEwen, 2008). There is a growing body of evidence that prenatal maternal distress adversely affects birth outcomes through these mechanistic pathways (Dunkel-Schetter, Gurung, Lobel, \& Wadhwa, 2001; Lobel, 1994; Lobel, Hamilton, \& Cannella, 2008), even when pregnancy is subjectively rated as only "somewhat" distressing (Lobel, Cannella, et al., 2008). Prenatal distress has been associated with negative perinatal outcomes including spontaneous abortion, structural malformations, preeclampsia, low birthweight, and preterm delivery (Dunkel-Schetter et al.; Zachariah, 2009) and with relatively long-term outcomes, including unfavorable neuroendocrine regulation and impaired neurobehavioral development in children (Mulder et al., 2002; Van den Bergh \& Marcoen, 2004; Van den Bergh, Mulder, Mennes, \& Glover, 2005). Therefore, because women experiencing pregnancy subsequent to miscarriage are at increased risk for prenatal distress, it is critical to understand the types, patterns, and moderators of distress experienced by this population in order to develop interventions to prevent undesirable stress-related outcomes.

\section{DISTRESS IN PREGNANCY SUBSEQUENT TO MISCARRIAGE}

According to descriptive studies of pregnancy following miscarriage and other types of perinatal loss, for some women the subsequent pregnancy is perceived as threatening (CôtéArsenault, 2007) and involves heightened vulnerability, anxiety, and guarded emotions related to uncertainty about its outcome (e.g., Côté-Arsenault \& Mahlangu, 1999; CôtéArsenault \& Marshall, 2000). Pregnancy anxiety and the appraisal of the subsequent pregnancy as threatening have been associated with the degree to which a mother assigns personhood to the deceased fetus (Côté-Arsenault, 2007; Côté-Arsenault \& Dombeck, 2001). Investigators who included comparison groups of women without a history of loss have found that women with a history of perinatal loss, including miscarriage, experience significantly greater state anxiety, pregnancy-specific anxiety, worry, depression, and less attachment to the subsequent pregnancy than women without a history of perinatal loss (Armstrong, 2002; Côté-Arsenault, 2003; Tsartsara \& Johnson, 2006). The most prevalent finding is that pregnancy-specific anxiety is higher in those with prior loss (Armstrong \& Hutti, 1998; Côté-Arsenault, 2003), but more generalized distress does not differ significantly between the groups (Côté-Arsenault, 2003; Franche \& Mikail, 1999; Theut, Pederson, Zaslow, \& Rabinovich, 1988).

Distress in pregnancy subsequent to miscarriage has been assessed at various time points. Some investigators have assessed prenatal distress retrospectively, during the postpartum period. Others, in investigations of distress at single time periods, have provided evidence that distress for women with prior loss is greater at early (Franche \& Mikail, 1999; Statham 
\& Green, 1994), mid- (Armstrong, 2002), and late pregnancy (Theut et al., 1988) when compared to women with no prior loss. However, it is likely that distress changes across this period of time. For instance, reaching anticipated prenatal milestones has been found to lower maternal anxiety in pregnancy subsequent to loss (Phipps, 1985). Reaching significant stages or time points in pregnancy, such as the period when previous loss occurred, also may affect distress (Côté-Arsenault \& Mahlangu, 1999). Among this growing group of researchers, only two teams have assessed distress at more than one time point (CôtéArsenault, 2007; Tsartsara \& Johnson, 2006), and both found that distress decreases significantly across pregnancy. A comparison group was included in one of these studies (Tsartsara \& Johnson). In this study, 10 women with a history of miscarriage were compared to 25 women without a history of miscarriage during the first and third trimester, although 5 of the women with a history of miscarriage and 7 of the women without a history of miscarriage were lost to follow-up at the third trimester assessment. Women with a history of miscarriage experienced greater pregnancy-specific anxiety at the first trimester assessment compared to their counterparts who had not experienced miscarriage. At the third trimester assessment, this difference was no longer significant, because anxiety had decreased more dramatically in the miscarriage group. However, these findings are tentative due to the greatly reduced sample size. In the only other study to include repeated assessments, pregnancy subsequent to perinatal loss was examined at three time periods across pregnancy (Côté-Arsenault, 2007). Psychological distress variables were examined once each trimester in 82 women with a history of perinatal loss. Côté-Arsenault (2007) found that pregnancy anxiety decreased significantly over the course of pregnancy. However, no comparison group was included.

\section{FACTORS INFLUENCING DISTRESS IN PREGNANCY SUBSEQUENT TO MISCARRIAGE}

Several factors might influence levels of distress in pregnancy subsequent to miscarriage. Women with a history of miscarriage tend to be older than women who have never experienced miscarriage (Nybo Andersen, Wohlfahrt, Christens, Olsen, \& Melbye, 2000). There is also evidence, although mixed, that women who have experienced miscarriage have lower socioeconomic status on average than women who have not experienced miscarriage (Price, 2006). These maternal characteristics, and not miscarriage history alone, may contribute to different emotional states during pregnancy for women who have and have not had a prior miscarriage. Thus, when examining the impact of miscarriage history on subsequent pregnancy, it is important to control for maternal characteristics that are correlated with miscarriage status. With rare exception (e.g., Tsartsara \& Johnson, 2006), investigators have not controlled for potential covariates. In addition, most samples have been composed of primarily White, married, educated women of middle to upper socioeconomic status (DeBackere et al., 2008). With one known exception (Hughes, Thurton, \& Evans, 1999), researchers have not included an ethnically and socioeconomically diverse sample when examining pregnancy subsequent to miscarriage and other types of perinatal loss.

Another factor that may influence psychological distress in pregnancy subsequent to miscarriage or perinatal loss is having a living child. Some researchers have found that having a living child may reduce distress either by providing confirmation to women that they can successfully carry a pregnancy to term, or by providing comfort because there is a child to love and nurture (Cuisinier, Janssen, de Graauw, Bakker, \& Hoogduin, 1996; Slade, 1994). Other researchers have not reached such conclusions. For example, in a study of emotional responses during the first year following miscarriage, Swanson et al. (2007) found that having a living child prior to miscarriage did not have a significant effect on emotional distress. Similarly, in another study of women with and without prior perinatal loss, the 
number of living children was not correlated significantly with either state or pregnancyspecific anxiety in women who were between 17 and 28 weeks gestation (Côté-Arsenault, 2003). In amore recent study, analysis of data collected only during the first trimester revealed that women with prior miscarriage reported elevated pregnancy-specific anxiety regardless of their parity status when compared to pregnant women without prior miscarriage (Tsartsara \& Johnson, 2006). The results of these studies suggest that having a living child may not buffer prenatal distress related to prior miscarriage (Côté-Arsenault, 2003; Tsartsara \& Johnson). Overall, investigations of the impact of living children on psychological distress in pregnancy subsequent to miscarriage have produced divergent results, which may be a result of methodological differences. For example, the samples of previous studies differ in regards to when the living child was born (e.g., the living child may have been born after or prior to the mother's experience with miscarriage).

Furthermore, in these studies, investigators examined the impact of parity status on distress in pregnancy subsequent to miscarriage at one time point only.

\section{The Current Study}

The purpose of the current study was to determine whether patterns of general and pregnancy-specific maternal distress, assessed across the three trimesters of pregnancy, were influenced by prior history of miscarriage, controlling for relevant demographics variables. Two hypotheses were tested:

Compared to pregnant women who have not experienced miscarriage, women with a history of miscarriage will experience:

Hypothesis 1: higher general distress (state anxiety) in the first, second, and third trimesters of pregnancy.

Hypothesis 2: higher pregnancy-specific distress in the first, second, and third trimesters of pregnancy.

Also explored was whether parity status moderates the impact of miscarriage status on generalized and pregnancy-specific distress.

The current study was designed to redress the limitations of previous research. Both a comparison group and longitudinal methods (assessment at the first, second, and third trimester) were used to examine prenatal distress in a socioeconomically and ethnically diverse sample of women. In addition, examining both general and pregnancy-specific distress allowed exploration of whether prior miscarriage and parity status affects these types of distress differently. Covariates of hypothesized relationships were identified and controlled.

\section{METHOD}

\section{Participants and Setting}

A prospective design was employed in the current study. The 363 participants in this study were part of a larger longitudinal study of psychosocial variables in pregnancy; data were collected from 1995 to 2000 (Lobel, Cannella et al., 2008). Eligibility for the larger study required participants to be at least 9 weeks of gestation at recruitment, to be at least 18 years old, and to speak English fluently, as the research was not designed to accommodate nonEnglish speakers or the unique issues surrounding adolescent pregnancy. The larger study was designed to include three interviews with women during pregnancy, with a minimum of 2 weeks between each participant's interviews. Participants were patients of a prenatal care facility in a large university-affiliated medical center located in the northeastern region of the United States. Approximately 606 (75\%) of the eligible women approached participated 
in the larger study. Women were included in this report if they completed at least $90 \%$ of the subset of variables examined in the present study. Attrition generally occurred as a result of participants declining interviews at particular visits, or leaving the prenatal care facility prior to completion of interviews. Women who were not included had lower household income, less education, and a greater number of previous pregnancies than the 363 women included in the study sample (all $p$ values < .05). Thirty-four percent of the sample was non-White, the average age at first interview was 27 years $(S D=5.9)$, and $85 \%$ of participants had at least a high school education. Table 1 includes a detailed demographic description of the participants.

\section{Measures}

Miscarriage-Because definitions of miscarriage vary (ACOG, 2009; Regan \& Rai, 2000; WHO, 2001), and because this study was an investigation of whether the experience of prior miscarriage affects emotions in pregnancy, a woman's perception of ever having a miscarriage was examined rather than miscarriage as defined by strict medical guidelines (e.g., spontaneous abortion). History of prior miscarriage was obtained, via self-report, at the first interview. Perception of miscarriage was assessed using the following item: "Have you ever had a miscarriage?" For the purposes of data analysis, an ever miscarriage variable was created; participants who responded no to this question were assigned to the no prior miscarriage group, and participants who responded yes to this question were assigned to the prior miscarriage group.

Demographics—Parity status, having a living child, was obtained by self-report. To assess parity status, participants were asked, "Do you have children?" Other demographic data, including information about age, marital status, obstetric history, and other sociodemographic variables (e.g., income, education), were obtained also by self-report at the first interview.

Distress-Two measures with established psychometric properties were used to assess psychological distress: The State Anxiety Subscale of the State-Trait Personality Inventory (STPI) and a revised version of the Prenatal Distress Questionnaire (PDQ). The 10-item STPI (Spielberger, 1995) was used to measure general distress. Respondents rate items such as "I feel nervous," "I am worried," and "I feel calm" (reverse-scored) on a 4-point scale ranging from 0 (not at all) to 3 (very much). Four items are reverse-scored. Responses for each interview were summed to create a total state anxiety score for each participant. The possible range of responses for total state anxiety is $0-30$. This measure has excellent validity and reliability (Spielberger, 1995) and is correlated highly with its longer parent measure, the State-Trait Anxiety Inventory (Spielberger, 1983). The internal consistency of the STPI in our study was high (Cronbach's $\alpha=.89, .91$, and .90 for first, second, and third interviews, respectively).

Pregnancy-specific distress was assessed because there is some evidence that this type of distress is more potent than distress of a general or nonspecific nature in women with a history of miscarriage (e.g., Côté-Arsenault, 2003) and that it is a stronger predictor of adverse birth outcomes than more general types of emotional distress (e.g., Lobel, Cannella, et al., 2008). A revised version of the PDQ (Lobel, Cannella, et al., 2008) was used to assess the degree to which the women were distressed by pregnancy-specific issues such as physical symptoms of pregnancy, changes in weight, paying for medical care, and changes in relationships. The original measure was designed for self-administration in midpregnancy (Yali \& Lobel, 1999). The measure was revised to be administered by an interviewer and repeated throughout pregnancy. The revised PDQ has excellent convergent and predictive validity (Lobel, Cannella, et al., 2008; Lobel, Hamilton, et al., 2008). It 
includes nine items that were repeated at the first, second, and third interviews. Participants were asked to indicate how concerned or worried they were about different aspects of pregnancy on a scale ranging from 0 (not at all) to 2 (very much). Responses for each interview were summed and divided by 9 to create an average PDQ score with a possible range of $0-2$. Internal consistency was acceptable (Cronbach's $\alpha=.60, .71$, and .81 for first, second, and third interviews, respectively). The modest internal consistency reflects the independence of some PDQ items, indicating that the measure differentiates concerns about specific aspects of pregnancy, as designed (Lobel, Cannella, et al., 2008).

\section{Procedure}

The study was approved by the Institutional Review Board. Eligible women were approached by trained research assistants as they awaited care in the prenatal care facility. Interested women were provided information about the purpose and nature of the study. Those meeting eligibility requirements and willing to participate provided their signatures to indicate informed consent to complete interviews and to allow access to their medical chart. To minimize participant burden, interviews were conducted in a private room at the prenatal care facility while participants awaited scheduled appointments.

\section{Data Analysis}

The three interviews were completed at various stages of pregnancy. For example, the first interview was completed as early as 9 weeks gestation for some participants and as late as 30 weeks for one woman. Not expecting anxiety and distress at 9 weeks gestation to be necessarily similar to that at 30 , outcomes were modeled according to gestational age, categorized into trimester.

As a result of the issues inherent to collecting data in a clinic setting (e.g., frequent rescheduling of appointments, no-shows, participant disinterest in completing an interview on a given appointment day), many women had more than one interview in the same trimester. The first interview occurred at an average gestational age of 17 weeks ( $S D=4.5$, range $=9-30$ ). Only 80 women (22\%) completed their first interview in their first trimester (less than 13 weeks gestation). Of the remaining women, 282 (77\%) completed their first interview in the second trimester (13-29 weeks), and one completed her first interview in her third trimester (30-41 weeks). The second interview was at an average gestational age of 26 weeks $(S D=3.8$, range $=20-35)$, and the third interview was at an average gestational age of 34 weeks $(S D=2.4$, range $=30-41)$.

A linear mixed model was used to assess the effects of past miscarriage on pregnancyspecific distress and state anxiety while allowing for within-subject correlations. The inclusion of trimester as a covariate in the model allowed the estimation of a separate effect of prior miscarriage for each trimester, and the repeated measures framework accounted for correlations among the three interviews within each subject. The sample size of 363 provided $87 \%$ power to detect a moderate effect size of .25 at each trimester. Models were fit using the MIXED procedure in SAS v9.2. Mixed models were used also to test the interaction between miscarriage status and parity status (living child[ren] or none) to determine whether having a living child buffers distress in women with a history of miscarriage.

\section{RESULTS}

Each of the 363 participants had three interviews. The sample sizes in Table 2 reflect the variable timing of the interviews. One participant did not have the gestational age recorded at her second interview, so only two of her interviews were included. Of the 1,088 total 
interviews, there were 80 in the first trimester, 559 in the second trimester, and 449 in the third trimester.

Chi-square analysis, Cochran-Armitage trend tests, and independent groups $t$-tests were used to compare the two miscarriage history groups on the sociodemographic variables in Table 1 . Women with a prior history of miscarriage were older, less likely to be currently employed, and had a lower total household income compared to women without a history of miscarriage (all $p \mathrm{~s}<.05$ ). These variables were included as covariates in the mixed models. In addition, women with previous miscarriage had a higher number of previous pregnancies, had given birth more times on average, and were more likely to have a living child. Parity status was included in the model to address whether having a living child buffers the effect of a miscarriage. Number of previous pregnancies and births were excluded from the model because of their close relationship to parity status.

\section{Distress and Miscarriage Status}

Means and standard deviations for anxiety and pregnancy-specific distress for participants and separately for participants with and without a history of miscarriage are presented in Table 2. State anxiety and pregnancy-specific distress were correlated $(r=.49, p<.001 ; r$ $=.49, p<.001 ; r=.44, p<.001 ;$ at the first, second, and third trimester, respectively).

State anxiety-Controlling for age, current employment status, and income, there was a significant relationship between previous miscarriage and state anxiety $(F[3,409]=7.93, p$ $<.001)$. Specifically, women who had a prior miscarriage had higher state anxiety in the second trimester $(t[424]=-3.21, p=.001)$ and third trimester $(t[424]=-4.07, p<.001)$ than women without prior miscarriage, but there was no statistically significant difference in the first trimester. State anxiety varied across the three trimesters $(F[2,409]=4.78, p=$. 009), with the modeled values of anxiety highest in the first trimester in both groups, then decreasing in the second and third trimester. Age and current employment status were statistically significant predictors of anxiety in the model, with anxiety being higher in younger women and those who were unemployed $(p<.001$ and $p=.03$, respectively). Income was not related significantly to anxiety. Modeled values of state anxiety for those with prior miscarriage versus those without are given in Figure 1, controlling for age, current employment status, and income equal to the sample median (27 years, no employment, and income $\$ 30,000-\$ 40,000)$.

Parity status and the interaction between miscarriage history and parity status were added to the mixed model to determine whether having a living child buffers the effect of miscarriage history on anxiety. In women without prior miscarriage, having a living child lowered their anxiety overall $(F[3,689]=3.14, p=.02)$. The magnitude of the effect was about 1.4 points on the STPI ( $0-3$ scale). This effect was consistent across the three trimesters, and was not seen in women with prior miscarriage $(F[3,689]=.70, p=.55)$. Age and current employment status were still significantly related to state anxiety, even after controlling for parity status.

Pregnancy-specific distress-There was no significant effect of miscarriage history on pregnancy-specific distress in early, mid-, or late pregnancy, controlling for age, current employment status, and income $(F[3,409]=1.18, p=.32)$. Distress did vary significantly across the three trimesters $(F[2,409]=22.73, p<.001)$. As depicted in Figure 2, both groups' pregnancy-specific distress scores decreased over time. None of the covariates in the model were significant predictors of pregnancy-specific distress.

Even though there was no significant effect of miscarriage history on distress, parity status and its interaction with miscarriage history were added to the mixed model to determine 
whether having a living child buffers pregnancy-specific distress in either group. Having a living child lowered distress in both the women with no miscarriage history $(F[3,689]=$ $4.84, p=.002)$ and the women with prior miscarriage $(F[3,689]=3.81, p=.01)$. The magnitude of the effect in both groups was about 0.1 points on the PDQ ( $0-2$ scale), and was consistent across the three trimesters.

\section{DISCUSSION}

\section{Summary of Findings}

The goal of the current study was to examine the influence of miscarriage history on patterns and magnitude of maternal distress during pregnancy. Compared with prior samples of pregnant women studied by our research team (e.g., Lobel, DeVincent, Kaminer, \& Meyer, 2000; Lobel, Dunkel-Schetter, \& Scrimshaw, 1992), participants in the present study had slightly higher state anxiety scores, and higher than population norms reported for nonpregnant women (Spielberger, 1983, 1995). Normative scores for the version of the PDQ used in this study to measure pregnancy specific distress have not yet been established; however, even low levels of distress (average PDQ item responses correspond to a rating between not at all and somewhat) predicted preterm delivery and health-impairing prenatal behaviors such as cigarette smoking in this sample (Lobel, Cannella, et al., 2008). In women both with and without prior miscarriage, state anxiety and pregnancy-specific distress were highest during the first trimester and decreased significantly throughout pregnancy. These findings corroborate those of investigators who used longitudinal methods to examine prenatal distress in women with and without prior miscarriage (Côté-Arsenault, 2007; Tsartsara \& Johnson, 2006).

\section{Influence of Miscarriage History on Maternal Distress During Pregnancy}

In the current study, women with prior miscarriage had higher state anxiety in the second and third trimesters of pregnancy when compared to women without prior miscarriage. This finding is similar to results reported by Hughes et al. (1999), who found higher state anxiety among women with a history of loss in the third trimester of a subsequent pregnancy when compared to women without prior loss.

Why would women with prior miscarriage experience first trimester levels of anxiety that are similar to women with no prior loss, but second and third trimester levels of anxiety that are significantly higher? Although pregnancy, in and of itself, is a stressful life event (see Geller, 2004; Lederman, 1995; Lobel, 1998), as pregnancy progresses emotional distress may decline less dramatically for women with prior miscarriage because their distress is related not only to the stressor of pregnancy but also to memories associated with the prior loss. Also, for these women, more may be at stake throughout the pregnancy than for women who have not experienced a prior loss.

There were no differences in pregnancy-specific distress when pregnant women with and without prior miscarriage were compared. Reports of pregnancy-specific distress may be similar for both groups of women, because the measure of this construct assesses symptoms and situations (e.g., low energy, medical bills, changes in body shape) that are relevant for all pregnant women at any stage of pregnancy (Lobel, Hamilton et al., 2008; Yali \& Lobel, 1999).

The findings that general distress (state anxiety) is more relevant than pregnancy-specific distress in women with a history of miscarriage is in contrast to findings of some prior studies. For example, Armstrong (2002) found both pregnancy-specific distress and nonspecific distress (depressive symptoms) to be higher in women with prior loss compared to those without prior loss. Other researchers have found that pregnancy-specific distress 
was more relevant than distress of a general or nonspecific nature in women with prior loss (Côté-Arsenault, 2003; Franche \& Mikail, 1999; Theut et al., 1988). There are psychometric issues that might help to explain these differences. The version of the PDQ used in this study differs from the instrument used to assess pregnancy-specific distress in other investigations of pregnancy following miscarriage, most commonly the Pregnancy Anxiety Scale (PAS; e.g., Côté-Arsenault, 2003). Despite the fact that the PDQ and the PAS are both intended to measure distress specific to pregnancy, the PAS has characteristics that are more similar to the state anxiety subscale of the Spielberger (1995) STPI, which was used in the current study to measure general distress. Both the PAS and the state anxiety instrument assess emotions such as worry, tension, and anxiety. However, unlike the state anxiety instrument, which is administered with instructions to complete the instrument items based on how interviewees feel "right now," the instructions for the PAS instruct interviewees to answer the questions as they specifically "think about this pregnancy." It is worthwhile to consider that when participants completed the state anxiety instrument, "right now" was during pregnancy and while the participant was waiting to be seen in a prenatal clinic. These contextual factors likely enhanced the salience of the current pregnancy. In other words, despite the fact that the STPI is designed to measure general (instead of pregnancy-specific) distress, when it is administered during pregnancy, participants' responses may have reflected emotions influenced by the pregnancy. This may have yielded responses that were pregnancy-specific. On the other hand, the PDQ is not used specifically to assess emotions, but instead to assess the degree to which specific "difficulties in pregnancy" (e.g., feeling tired, paying for medical care, symptoms of pregnancy) are "uncomfortable or upsetting." Another difference between the PAS and the PDQ is that the PAS assesses fear of pregnancy loss, and the version of the PDQ used in this study does not. These comparisons demonstrate that caution should be used when comparing findings across studies. Varying degrees of difference may exist between measures, despite the shared labels of constructs (e.g., "pregnancy-specific distress") that instruments are designed to measure. In light of these methodological challenges, it may be useful to continue to assess both pregnancy-specific and general distress in clinical practice to obtain the most comprehensive understanding of a woman's subjective experience of distress during pregnancy.

Differences in sample characteristics also may explain why the findings of the current study depart somewhat from previous research. Participants in prior studies were mostly White, highly educated, middle- to upper-class women. The results of the current study suggest that among socioeconomically and ethnically diverse women, perhaps general anxiety, and not pregnancy-specific distress, is more likely to be triggered by prior perinatal experience because other life stressors impinge on women with fewer educational, social, and economic resources and affect the way they cope with their loss (Hamilton \& Lobel, 2008).

\section{Do Living Children Buffer Prenatal Distress Subsequent to Miscarriage?}

Having a living child was not associated with lower state anxiety in women with a history of miscarriage; experience with miscarriage influenced this type of distress regardless of parity status. This finding partially corroborates previous research (Côté-Arsenault, 2003; Tsartsara \& Johnson, 2006) and suggests that despite a woman's prior successful experience with pregnancy, a previous miscarriage influences her emotional state. A living child is not a replacement for the child who was lost and does not buffer prenatal distress subsequent to miscarriage. On the other hand, having a living child was associated with lower pregnancyspecific distress in both women with and without a history of miscarriage. These results make sense, because the instrument used to measure pregnancy-specific distress assesses the life challenges that are typical during pregnancy. Women with living children have had previous successful pregnancies, so it is reasonable that they may be less upset by these pregnancy-related transitions or adjustments. 


\section{The Impact of Covariates}

The current study was one of the first to include covariates when examining the impact of miscarriage history on prenatal distress during a subsequent pregnancy. Tsartsara and Johnson (2006) examined the impact of demographic and reproductive variables on pregnancy-specific anxiety in women with and without prior miscarriage. Using this methodology, these researchers found that only parity had a significant effect on women's levels of pregnancy-specific anxiety. Other demographic measures (e.g., maternal age, marital status, gestation age, pregnancy planning, gravidity, parity, having an ultrasound scan during index pregnancy) did not influence pregnancy-specific anxiety. In the current study, a different analytic method was used to examine the influence of potential covariates on the relationship between miscarriage status and prenatal distress in subsequent pregnancy. The goal was to ensure that demographic factors were not confounding the effects of miscarriage on prenatal distress. Therefore, the relationships among specific sociodemographic variables (Table 1) and miscarriage status were examined by comparing the demographic profile of women with and without a history of miscarriage.

Women with a history of miscarriage were older, more likely to be unemployed, and had lower total household income than women without a history of miscarriage. These variables were included as covariates in the analysis of relationships between miscarriage status and prenatal distress. When these variables were included, younger women and those who were unemployed reported higher anxiety. Such findings underscore the importance of examining sociodemographic factors and other maternal characteristics that are associated with miscarriage, as these factors may affect the impact of miscarriage history on women's emotional state. The inverse relationship between age and psychological distress is consistent with previous research (Giurgescu, Penckofer, Maurer, \& Bryant, 2006; Green, Kafetsios, Statham, \& Snowdon, 2003).

Higher anxiety in younger women may be related to the fact that they have fewer life experiences that would enhance confidence or buffer feelings of fear, worry, or tension associated with the major life transition of pregnancy (Sepa, Frodi, \& Ludvigsson, 2004). It is not clear why participants with prior miscarriage were more likely to be unemployed or why unemployment was associated with higher anxiety, especially as lower income was not related to higher anxiety.

It is possible that an unmeasured variable associated with employment status explains this finding. In the future, researchers could examine factors that contribute to unemployment. For instance, is unemployment voluntary or involuntary? Women with prior miscarriage may be more likely to choose to be unemployed during a subsequent pregnancy to avoid work-related emotional or physical strain. Alternatively, it is possible that unemployment limits stress-buffering social support resources that could be beneficial during pregnancy (Misra, Guyer, \& Allston, 2003; Reime, Jacob, \& Wenzlaff, 2009). Future research may provide greater insight into these issues.

\section{Implications and Limitations}

The current study was limited by a lack of detail relevant to the miscarriage, such as number of previous miscarriages, gestation of previous miscarriage, time between miscarriage and current pregnancy, and post-miscarriage distress and adjustment. This information would provide a more complete understanding of the effects of the miscarriage on feelings of distress during the subsequent pregnancy. Future researchers might include such data, as well as longitudinal methods to investigate how the timing of successful pregnancies in relation to a miscarriage contributes to women's prenatal emotions. This research also could build upon Statham and Green's (1994) findings that when women have successful 
pregnancies after a pregnancy loss (and not before), having a living child reduces distress early in subsequent pregnancy.

Women included in this study were socioeconomically and ethnically diverse, but those not included in analyses because of attrition were less likely to be married and had a significantly greater number of previous pregnancies, lower income, and less education. Additional studies are needed to investigate the effect that tangible, social, and intrapersonal resources have on distress in pregnancies following miscarriage. Furthermore, future researchers might attempt to examine what mediates the impact of perinatal loss on distress to develop tailored interventions.

Results of our study demonstrate that including methodological strengths such as controls for covariates and assessment of multiple types of distress at multiple time points during pregnancy provides important information about patterns and levels of distress in women who experience pregnancy subsequent to miscarriage. In future research, use of existing prenatal distress instruments (e.g., PAS, PDQ, and State Anxiety) may enhance comparability of results across studies. Furthermore, it may be useful to investigate the degree of overlap among these measures.

\section{Conclusions}

A significant body of literature shows that women with prior miscarriage benefit from emotional support (Côté-Arsenault \& Marshall, 2000; Swanson, 1999b; Zachariah, 2009). Future researchers could investigate the potential benefits of supportive interventions implemented during subsequent pregnancy, and particularly at mid- and late pregnancy, when women with prior miscarriage in this study were found to have higher distress compared to women with no prior miscarriage. In addition, miscarriage has an impact on maternal anxiety during subsequent pregnancy, despite the presence of other children. These findings can be used by healthcare providers, family members, and friends to guide the support they provide to pregnant women who have experienced a miscarriage.

\section{Acknowledgments}

This study was funded by NIH Grant NR03443 (Lobel, PI). Cheryl L. Woods-Giscombé received support from NIH/NINR Grant T32NR007091, the NIH Loan Repayment Program (NCMHD), and the Substance Abuse and Mental Health Services Administration Minority Fellowship Program at the American Nurses Association during work on this manuscript. We are grateful to Anne Moyer, Jennifer Leeman, and Chris Weisen for helpful comments on earlier versions of this manuscript.

\section{REFERENCES}

American College of Obstetricians and Gynecologists. Repeated miscarriage (ACOG Education Pamphlet AP100). Washington, DC: Author; 2009.

Armstrong DS. Emotional distress and prenatal attachment in pregnancy after perinatal loss. JOGNNJournal of Nursing Scholarship. 2002; 34:339-345.

Armstrong D, Hutti M. Pregnancy after perinatal loss: The relationship between anxiety and prenatal attachment. Journal of Obstetric, Gynecologic, and Neonatal Nursing. 1998; 27:183-189.

Côté-Arsenault D. The influence of perinatal loss on anxiety in multigravidas. Journal of Obstetric, Gynecologic, and Neonatal Nursing. 2003; 32:623-629.

Côté-Arsenault D. Threat appraisal, coping, and emotions across pregnancy subsequent to perinatal loss. Nursing Research. 2007; 56:108-116. [PubMed: 17356441]

Côté-Arsenault D, Dombeck MT. Maternal assignment of fetal personhood to a previous pregnancy loss: Relationship to anxiety in the current pregnancy. Health Care for Women International. 2001; 22:649-665. [PubMed: 12141842] 
Côté-Arsenault D, Mahlangu N. Impact of perinatal loss on the subsequent pregnancy and self: Women's experiences. Journal of Obstetric, Gynecologic, and Neonatal Nursing. 1999; 28:274282.

Côté-Arsenault D, Marshall R. One foot in-One foot out: Weathering the storm of pregnancy after perinatal loss. Research in Nursing \& Health. 2000; 23:473-485. [PubMed: 11130606]

Cuisinier M, Janssen H, de Graauw C, Bakker S, Hoogduin C. Pregnancy following miscarriage: Course of grief and some determining factors. Journal of Psychosomatic Obstetrics and Gynaecology. 1996; 17:168-174. [PubMed: 8892163]

DeBackere KJ, Hill PD, Kavanaugh KL. The parental experience of pregnancy after perinatal loss. Journal of Obstetric Gynecologic, \& Neonatal Nursing. 2008; 37:525-537.

DeLuca RS, Lobel M. Conception, commitment, and health behavior practices in medically high-risk pregnant women. Women's Health. 1995; 1:257-271.

Dunkel-Schetter, C.; Gurung, RAR.; Lobel, M.; Wadhwa, PD. Stress processes in pregnancy and birth: Psychological, biological, and sociocultural influences. In: Baum, A.; Revenson, TA.; Singer, JE., editors. Handbook of health psychology. Hillsdale, NJ: Erlbaum; 2001. p. 495-518.

Franche RL, Mikail SF. The impact of perinatal loss on adjustment to subsequent pregnancy. Social Science \& Medicine. 1999; 48:1613-1623. [PubMed: 10400261]

Frost M, Condon JT. The psychological sequelae of miscarriage: A critical review of the literature. The Australian and New Zealand Journal of Psychiatry. 1996; 30:54-62. [PubMed: 8724327]

Geller PA. Pregnancy as a stressful life event. CNS Spectrums. 2004; 9:188-197. [PubMed: 14999159]

Geller PA, Kerns D, Klier CM. Anxiety following miscarriage and the subsequent pregnancy: A review of the literature and future directions. Journal of Psychosomatic Research. 2004; 56:35-45. [PubMed: 14987962]

Giurgescu C, Penckofer S, Maurer MC, Bryant FB. Impact of uncertainty, social support, and prenatal coping on the psychological well-being of high-risk pregnant women. Nursing Research. 2006; 55:356-365. [PubMed: 16980836]

Green JM, Kafetsios K, Statham HE, Snowdon CM. Factor structure, validity and reliability of the Cambridge Worry Scale in a pregnant population. Journal of Health Psychology. 2003; 8:753-764. [PubMed: 14670208]

Hamilton JG, Lobel M. Types, patterns, and predictors of coping with stress during pregnancy: Examination of the Revised Prenatal Coping Inventory in a diverse sample. Journal of Psychosomatic Obstetrics and Gynaecology. 2008; 29:97-104. [PubMed: 18484440]

Hughes PM, Thurton P, Evans CD. Stillbirth as risk factor for depression and anxiety in the subsequent pregnancy: Cohort study. British Medical Journal. 1999; 318:1721-1724. [PubMed: 10381705]

Lederman RP. Relationship of anxiety, stress, and psychosocial development to reproductive health. Behavioral Medicine. 1995; 21:101-112. [PubMed: 8789646]

Lobel M. Conceptualizations, measurement, and effects of prenatal maternal stress on birth outcomes. Journal of Behavioral Medicine. 1994; 17:225-272. [PubMed: 7932680]

Lobel, M. Pregnancy and mental health. In: Friedman, H., editor. Encyclopedia of mental health. San Diego, CA: Academic Press; 1998. p. 229-238.

Lobel M, Cannella DL, Graham JE, DeVincent C, Schneider J, Meyer BA. Pregnancy-specific stress, prenatal health behaviors, and birth outcomes. Health Psychology. 2008; 27:604-615. [PubMed: 18823187]

Lobel M, DeVincent CJ, Kaminer A, Meyer BA. The impact of prenatal maternal stress and optimistic disposition on birth outcomes in medically high-risk women. Health Psychology. 2000; 19:544553. [PubMed: 11129357]

Lobel M, Dunkel-Schetter C, Scrimshaw SCM. Prenatal maternal stress and prematurity: A prospective study of socioeconomically disadvantaged women. Health Psychology. 1992; 11:3240. [PubMed: 1559532]

Lobel M, Hamilton JG, Cannella DL. Psychosocial perspectives on pregnancy: Prenatal maternal stress and coping. Social \& Personality Psychology Compass. 2008; 2:1600-1623. 
McEwen BS. Central effects of stress hormones in health and disease: Understanding the protective and damaging effects of stress and stress mediators. European Journal of Pharmacology. 2008; 583:174-185. [PubMed: 18282566]

Misra DP, Guyer BG, Allston A. Integrated perinatal health framework: A multiple determinants model with a life span approach. American Journal of Preventive Medicine. 2003; 25:65-75. [PubMed: 12818312]

Mulder EJH, Robles de Medina PG, Huizink AC, Van den Bergh BRH, Buitelaar JK, Visser GHA. Prenatal maternal stress: Effects on pregnancy and the (unborn) child. Early Human Development. 2002; 70:3-14. [PubMed: 12441200]

Nybo Andersen AM, Wohlfahrt J, Christens P, Olsen J, Melbye M. Maternal age and fetal loss: Population based register linkage study. British Medical Journal. 2000; 320:1708-1712. [PubMed: 10864550]

Phipps S. The subsequent pregnancy after stillbirth: Anticipatory parenthood in the face of uncertainty. International Journal of Psychiatry in Medicine. 1985; 15:243-264. [PubMed: 4066164]

Price SK. Prevalence and correlates of pregnancy loss history in a national sample of children and families. Maternal and Child Health Journal. 2006; 10:489-500. [PubMed: 16802189]

Reime B, Jacob C, Wenzlaff P. Is parental unemployment related to an increased risk for stillbirths? Journal of Public Health. 2009; 17:363-369.

Regan L, Rai R. Epidemiology and the medical causes of miscarriage. Bailliere's Best Practice \& Research. Clinical Obstetrics \& Gynaecology. 2000; 14:839-854. [PubMed: 11023804]

Sepa A, Frodi A, Ludvigsson J. Psychosocial correlates of parenting stress, lack of support and lack of confidence/security. Scandinavian Journal of Psychology. 2004; 45:169-179. [PubMed: 15016271]

Slade P. Predicting the psychological impact of miscarriage. Journal of Reproductive and Infant Psychology. 1994; 12:5-16.

Spielberger, CD. Manual for the state-trait anxiety inventory. Palo Alto, CA: Consulting Psychologists Press; 1983.

Spielberger, CD. Preliminary manual for the state-trait personality inventory. Tampa, FL: Center for Research in Behavioral Medicine and Health Psychology, University of South Florida; 1995.

Statham H, Green JM. The effects of miscarriage and other 'unsuccessful' pregnancies on feelings early in a subsequent pregnancy. Journal of Reproductive and Infant Psychology. 1994; 12:45-54.

Swanson KM. Effects of caring, measurement, and time on miscarriage impact and women's wellbeing. Nursing Research. 1999a; 48:288-298. [PubMed: 10571496]

Swanson KM. Research-based practice with women who have had miscarriages. Image: The Journal of Nursing Scholarship. 1999b; 31:339-345. [PubMed: 10628100]

Swanson KM, Connor S, Jolley SN, Pettinato M, Wang TJ. Contexts and evolution of women's responses to miscarriage during the first year after loss. Research in Nursing \& Health. 2007; 30:2-16. [PubMed: 17243104]

Theut SK, Pederson FA, Zaslow MJ, Rabinovich BA. Pregnancy subsequent to perinatal loss: Parental anxiety and depression. Journal of the American Academy of Child and Adolescent Psychiatry. 1988; 27:289-292. [PubMed: 3379012]

Tsartsara E, Johnson MP. The impact of miscarriage on women's pregnancy-specific anxiety and feelings of prenatal maternal-fetal attachment during the course of a subsequent pregnancy: An exploratory follow-up study. Journal of Psychosomatic Obstetrics and Gynaecology. 2006; 27:173-182. [PubMed: 17214452]

Tulandi, T.; Al-Fozan, HM. Spontaneous abortion: Risk factors, etiology, clinical manifestations, and diagnostic evaluation. In: Levine, D.; Barbieri, RL., editors. UpToDate clinical reference library. Waltham, MA: Wolters Kluwer; 2009. from http://www.uptodate.com/patients/content/topic.do?topicKey= treIIBdkibqkdbS

Van den Bergh BRH, Marcoen A. High antenatal maternal anxiety is related to ADHD symptoms, externalizing problems, and anxiety in 8- and 9-year-olds. Child Development. 2004; 75:10851097. [PubMed: 15260866] 
Van den Bergh BRH, Mulder EJ, Mennes M, Glover V. Antenatal maternal anxiety and stress and the neurobehavioural development of the fetus and child: Links and possible mechanisms. A review. Neuroscience and Biobehavioral Reviews. 2005; 29:237-258. [PubMed: 15811496]

World Health Organization. Definitions and indicators in family planning, maternal \& child health, and reproductive health. Geneva, Switzerland: WHO Press; 2001.

Yali AM, Lobel M. Coping and distress in pregnancy: An investigation of medically high risk women. Journal of Psychosomatic Obstetrics and Gynecology. 1999; 20:39-52. [PubMed: 10212886]

Zachariah R. Social support, life stress, and anxiety as predictors of pregnancy complications in lowincome women. Research in Nursing \& Health. 2009; 32:391-404. [PubMed: 19434649] 


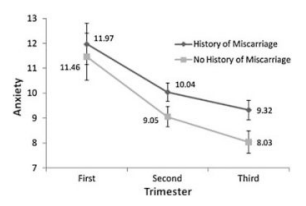

FIGURE 1.

Mixed model estimates of anxiety by trimester, with and without history of miscarriage controlling for age, income, and current employment status. Error bars indicate \pm 1 standard error. 


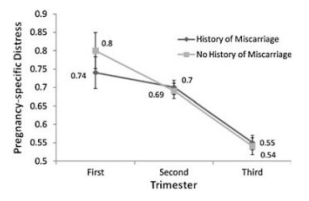

FIGURE 2.

Mixed model estimates of pregnancy-specific distress by trimester with and without history of miscarriage controlling for age, income, and current employment status. Error bars indicate \pm 1 standard error. 
Table 1

Comparison of Women With and Without Previous Miscarriage

\begin{tabular}{|c|c|c|}
\hline & $\begin{array}{r}\text { Previous Miscarriage } \\
(n=113)\end{array}$ & $\begin{array}{r}\text { No Previous Miscarriage } \\
\qquad(n=250)\end{array}$ \\
\hline Age, $M(S D)^{* *}$ & $28.2(5.68)$ & $26.5(5.88)$ \\
\hline \multicolumn{3}{|l|}{ Race, $n(\%)$} \\
\hline White & $64(56.6)$ & $174(69.6)$ \\
\hline Black & $17(15.0)$ & $27(10.8)$ \\
\hline Latina & $14(12.4)$ & $27(10.8)$ \\
\hline Asian or Pacific Islander & $2(1.80)$ & $5(2.00)$ \\
\hline Native American & $1(0.90)$ & $1(0.40)$ \\
\hline Multiethnic & $14(12.4)$ & $17(6.80)$ \\
\hline \multicolumn{3}{|l|}{ Living arrangements, $n(\%)$} \\
\hline Living with baby's father & $78(69.0)$ & $168(67.2)$ \\
\hline Living with family & $22(19.5)$ & $53(21.2)$ \\
\hline Living with friends & $2(1.80)$ & $2(0.80)$ \\
\hline Living alone & $9(8.00)$ & $10(4.00)$ \\
\hline Other & $2(1.80)$ & $17(6.80)$ \\
\hline \multicolumn{3}{|l|}{ Education, $n(\%)$} \\
\hline Junior High School & $3(2.7)$ & $1(0.40)$ \\
\hline Some High School & $19(16.8)$ & $30(12.2)$ \\
\hline Graduated High School & $39(34.5)$ & $97(39.3)$ \\
\hline Some College & $42(37.2)$ & $94(38.1)$ \\
\hline Graduated College & $9(8.0)$ & $19(7.7)$ \\
\hline Graduate Degree & $1(0.9)$ & $6(2.4)$ \\
\hline \multicolumn{3}{|l|}{ Annual household income, $n(\%)^{*}$} \\
\hline Under $\$ 10,000$ & $20(18.0)$ & $29(12.1)$ \\
\hline$\$ 10,000-\$ 20,000$ & $34(30.6)$ & $54(22.5)$ \\
\hline$\$ 20,000-\$ 30,000$ & 24 (21.6) & $67(27.9)$ \\
\hline$\$ 30,000-\$ 40,000$ & $14(12.6)$ & $36(15.0)$ \\
\hline$\$ 40,000-\$ 50,000$ & $10(9.0)$ & $24(10.0)$ \\
\hline More than $\$ 50,000$ & $9(8.1)$ & $30(12.5)$ \\
\hline Pregnant previously, $n(\%){ }^{* * *} \mathrm{CA}=\mathrm{L}>113(100)$ & $177(70.8)$ & \\
\hline Previous pregnancies, $M(S D)^{* * *}$ & $3.76(2.32)$ & $1.44(1.43)$ \\
\hline Times given birth, $M(S D)^{* * *}$ & $1.52(1.45)$ & $0.95(1.10)$ \\
\hline Has living child (parity), $n(\%)^{* * *}$ & $83(73.5)$ & $135(54)$ \\
\hline Planned pregnancy, $n(\%)$ & $37.0(32.7)$ & $61(24.4)$ \\
\hline \multicolumn{3}{|l|}{ Time to get pregnant, $n(\%)$} \\
\hline NA & $76(67.3)$ & $189(75.6)$ \\
\hline Less than 6 months & $23(20.4)$ & $44(17.6)$ \\
\hline $6-11$ months & $4(3.50)$ & $8(3.20)$ \\
\hline $1-2$ years & $6(5.30)$ & $5(2.00)$ \\
\hline
\end{tabular}




\begin{tabular}{|c|c|c|}
\hline & $\begin{array}{r}\text { Previous Miscarriage } \\
(n=113)\end{array}$ & $\begin{array}{r}\text { No Previous Miscarriage } \\
\qquad(n=250)\end{array}$ \\
\hline Greater than 2 years & $2(1.80)$ & $3(1.20)$ \\
\hline Unsure & $2(1.8)$ & $1(0.40)$ \\
\hline \multicolumn{3}{|c|}{ Pre-pregnancy employment, $n(\%)$} \\
\hline Full-time & $42(37.2)$ & $114(45.6)$ \\
\hline Part-time & $31(27.4)$ & $76(30.4)$ \\
\hline Unemployed & $40(35.4)$ & $60(24.0)$ \\
\hline \multicolumn{3}{|c|}{ Current employment, $n(\%){ }^{*}$} \\
\hline Full-time & $18(15.9)$ & $55(22.0)$ \\
\hline Part-time & $24(21.2)$ & $78(31.2)$ \\
\hline Unemployed & $71(62.8)$ & $117(46.8)$ \\
\hline \multicolumn{3}{|c|}{ Gestational age (weeks), $M(S D)$} \\
\hline First assessment & $17.1(4.56)$ & $16.7(4.46)$ \\
\hline Second assessment & $26.0(3.64)$ & $25.9(3.84)$ \\
\hline Third assessment & $34.1(2.37)$ & $33.9(2.40)$ \\
\hline \multicolumn{3}{|l|}{$*<.05}$. \\
\hline \multicolumn{3}{|l|}{$\begin{array}{r}* * \\
\quad p<.01 .\end{array}$} \\
\hline$* * * x<.001$. & & \\
\hline
\end{tabular}


Table 2

Means and Standard Deviations for Anxiety and Pregnancy-Specific Distress

\begin{tabular}{|c|c|c|c|c|c|}
\hline \multirow[b]{2}{*}{ Variable } & \multirow[b]{2}{*}{ Trimester } & \multirow[b]{2}{*}{$\begin{array}{l}\text { Number of Observations (Number } \\
\text { From Participants With Miscarriage) }\end{array}$} & \multicolumn{3}{|c|}{$M(S D)$} \\
\hline & & & All Participants & $\begin{array}{c}\text { History of } \\
\text { Miscarriage }\end{array}$ & $\begin{array}{l}\text { No History of } \\
\text { Miscarriage }\end{array}$ \\
\hline \multirow[t]{3}{*}{ Anxiety ${ }^{a}$} & First & $79(24)$ & $11.0(6.93)$ & $11.8(7.18)$ & $10.7(6.86)$ \\
\hline & Second & 559 (177) & $9.2(6.92)$ & $10.4(7.30)$ & $8.7(6.67)$ \\
\hline & Third & 449 (138) & $8.4(6.41)$ & $10.1(7.54)$ & $7.6(5.69)$ \\
\hline \multirow[t]{3}{*}{ Pregnancy-specific distress $b$} & First & $79(24)$ & $.74(0.34)$ & $.66(.38)$ & $.77(.32)$ \\
\hline & Second & $559(177)$ & $.68(0.35)$ & $.69(.36)$ & $.67(.35)$ \\
\hline & Third & 449 (138) & $.53(0.33)$ & $.55(.34)$ & $.52(.32)$ \\
\hline
\end{tabular}

Notes: Means and standard deviations for second and third trimesters include multiple observations from many subjects. They are presented in order to describe the pattern of anxiety and distress across pregnancy, but should not be used as direct tests of mean difference or in meta-analyses due to the nonindependence of subjects.

${ }^{a}$ Possible range of scores for anxiety was 0-30.

${ }^{b}$ Possible range of scores for pregnancy-specific distress was 0-2. 